\title{
BMJ Open Recognition of anxiety disorders in children: a cross-sectional vignette-based survey among general practitioners
}

\author{
Semiha Aydin (1) , ${ }^{1,2,3}$ Mathilde R Crone, ${ }^{1}$ Bart M Siebelink, ${ }^{3}$ \\ Robert R J M Vermeiren, ${ }^{3,4}$ Mattijs E Numans, ${ }^{1}$ P Michiel Westenberg ${ }^{2}$
}

To cite: Aydin S, Crone MR, Siebelink BM, et al. Recognition of anxiety disorders in children: a cross-sectional vignettebased survey among general practitioners. BMJ Open 2020;10:e035799. doi:10.1136/ bmjopen-2019-035799

- Prepublication history and additional material for this paper are available online. To view these files, please visit the journal online (http://dx.doi. org/10.1136/bmjopen-2019035799).

Received 15 November 2019

Revised 10 March 2020

Accepted 13 March 2020

Check for updates

(C) Author(s) (or their employer(s)) 2020. Re-use permitted under CC BY-NC. No commercial re-use. See rights and permissions. Published by BMJ.

${ }^{1}$ Department of Public Health and Primary Care, Leiden University Medical Center, Leiden, Zuid-Holland, The Netherlands

${ }^{2}$ Department of Developmental and Educational Psychology,

Leiden University, Leiden, ZuidHolland, The Netherlands

${ }^{3}$ Department of Child and Adolescent Psychiatry, CuriumLUMC, Leiden, Zuid-Holland, The Netherlands

${ }^{4}$ Youz, Rotterdam, Zuid-Holland, The Netherlands

Correspondence to

Semiha Aydin;

s.aydin@fsw.leidenuniv.nl

\section{ABSTRACT}

Objective To quantify general practitioners' (GPS) sensitivity to anxiety disorders (ADs) when confronted with the range of symptoms common to children with ADs. Also, to explore GPs' conscious preferences and implicit tendencies for referral of children with ADs to mental healthcare.

Design and setting In a cross-sectional vignette-based survey, all attendees of a Dutch continuing medical education conference for primary care physicians were presented with subtitled audio fragments of five vignettes that we constructed to mimic symptom presentation of children with ADs in general practice. We asked attendees to select per vignette the most plausible diagnoses and most adequate referral option, and for their general referral preferences when they suspect each of the most common mental health problems.

Participants A sample of $229 \mathrm{GPs}$, resulting in a total of 1128 vignette evaluations.

Main outcome measure GPs' selection rate of ADs in the five vignettes compared with a benchmark provided by mental health professionals (MHPs).

Results Overall, recognition of ADs was less likely in GPs compared with MHPs (OR=0.26, $95 \% \mathrm{Cl} 0.15$ to 0.46 ). GPs varied in their recognition of anxiety, with $44.1 \%$ not once selecting anxiety as the probable presenting problem. When asked explicitly, $63.9 \%$ of the GPs reported that they would refer a child to mental healthcare when they suspect probable ADs. By contrast, only $12.0 \%$ of the GPs who recognised anxiety in the vignettes actually selected that referral option.

Conclusion A significant fraction of GPs did not notice the depicted symptoms as anxiety. Despite the widespread prevalence of ADs, GPs seem to overlook anxiety already in their early diagnostic opinion. Improving GPs' familiarity with initial symptom presentation, ADs' baserate, relevance and impact yields potential for timely recognition.

\section{INTRODUCTION}

Unnoticed anxiety disorders (ADs) have an important impact on human development and well-being. Only one in ten children with ADs, including those with subthreshold levels of severity, will be free of any mental health problem later in life. ${ }^{1-7}$ With estimated prevalence rates of up to one in four, $\mathrm{ADs}$ are

\section{Strengths and limitations of this study}

- This is the first study relating general practitioners (GPs) interpretation of the broad range of presenting problems in daily practice to the timely recognition of anxiety disorders in children.

- The vignette-based design enabled exclusion of putative external effects of the complex clinical reality, and the presentation of the vignettes through subtitled audio fragments the isolation of GPs' sensitivity to pick up on the signs of anxiety.

- While the verbal presentation of five vignettes to every participant in the same order was substantial to investigate overall recognition, the results are only exploratory on the level of the individual vignettes and various symptoms.

- The predefined response options limited access to GPs' considerations; mixed-methods studies might enhance in-depth understanding of their interpretations and decision-making process.

the most common mental health issue over the lifespan. ${ }^{8-10}$ Fortunately, treatment helps to mitigate risks and adversities. ${ }^{11-14}$ Based on their gate-keeper role, approachability and their continuous care relationship with families, general practitioners (GPs) are in a unique position to recognise $\mathrm{ADs}$ characterised by an early age of onset, a chronic or relapsing-remitting course, somatic manifestations and comorbidities. ${ }^{15-20}$ Although around $70 \%$ of children and adolescents consult their GP more than twice a year, ${ }^{21-24}$ studies in primary healthcare indicate that over two-thirds of children with ADs go unnoticed. ${ }^{25-28}$

Recognition is especially problematic in early onset, less severe and less explicit disorders. ${ }^{28-31}$ Interestingly, those factors are inherent to anxiety, including the covert nature of its core symptoms, its gradual development, fluctuating course and the wide variety of accompanying symptoms that are not prototypic for anxiety. ${ }^{3}$ ADs are tied to problems as temper tantrums, 
control neediness, withdrawn behaviour, difficulties in connecting with others, poor concentration and physical complaints. ${ }^{32}$ Each of these indicators are seemingly unrelated and may not equally impact a child's situation, increasing the chances that professionals fail to appreciate them as a relevant or important sign. These symptoms also mimic and co-occur with other mental health disorders and contextual problems. Parents and teachers show differences in recognition of various symptoms and tend to underdetect or misattribute some more than the others. ${ }^{33-36}$

Given the limited time available to understand and act on the breadth of disclosed problems, a GP's first interpretation of the presented problems might be pivotal in successfully navigating a consultation and recognising anxiety in children. The decision-making literature highlights the importance of the first diagnostic opinion and suggests that amendments after this point are often insufficient ('anchoring bias'). ${ }^{37}$ However, studies that relate recognition to the variety of symptoms are scarce, and to our knowledge, no study has investigated in how far physicians even consider anxiety as the probable factor underlying the reason of consult. To address this, we presented GPs with mixed-anxiety vignettes. That is, vignettes that capture the variety and indefinite nature of early symptom presentation in general practice and do not solely describe specific mental health disorders as demarcated in psychiatric nosologies. Notwithstanding that we created the vignettes to suggest a probable $\mathrm{AD}$, we hypothesised that GPs would not proportionately cite anxiety as the underlying cause of the presented symptoms. In addition to the question how GPs would interpret and refer the described cases, we also asked how they generally tend to manage cases when they suspect specific mental health problems in practice. Based on literature suggesting that ADs might be evaluated as relatively benign, ${ }^{38}$ we hypothesised that GPs would be less likely to report that they refer children with suspected anxiety to mental healthcare (MHC) than children with suspected developmental, behavioural or mood problems.

\section{METHODS}

\section{Setting and design}

The study was conducted during the yearly 2-day continuing medical education conference 'Boerhaave Progress and Practice ${ }^{, 39}$ for primary healthcare physicians (Leiden, The Netherlands, 14 December 2018). Each year, a range of somatic and mental health topics are covered by specialists within 25 min time slots. During one of those slots, we presented the survey supported by PowerPoint slides that additionally carried embedded subtitled audio fragments of the vignettes (60-64s each). A total of 275 GPs were registered. Attendees were seated with a device that digitally recorded their anonymous responses. As such, data collection was not subject to Dutch law governing medical research involving human subjects, nor to European law on general data protection and privacy.

\section{Procedure and measurement}

Attendees were introduced with (1) that they would hear five case descriptions of children with psychosocial problems in the form of a report resulting from a few consultations, and would see three recurring questions that reflect their daily reality concerning what the condition might be and what could be done about it. (2) They were asked to base their considerations on the available information only. We aimed to use gender-neutral names for the vignettes to prevent that gender confounds decisionmaking. In an independent coding of 25 gender-neutral names by six coders, at most three names were labelled by half of the coders as gender-neutral. Given this low agreement and taking into account discrepancies in literature on gender differences and recognition, ${ }^{29}{ }^{36}$ we mentioned (3) that the named cases were intentionally gender-neutral. Attendees were also informed (4) that by responding they voluntarily and anonymously agreed to analysis of their responses for scientific purposes. Preceding the first vignette, the recurring questions were verbally introduced and shown (table 1 ). Thereafter, the questions were shown sequentially, each with a progress bar displaying the response time set to a maximum of $10 \mathrm{~s}$.

\section{Vignette development}

In order to quantify to what extend GPs consider anxiety in their early diagnostic opinion, we constructed vignettes that capture various symptoms (online supplementary file A). Guided by knowledge of the clinical presentation of ADs and the prevalence of common mental health disorders, we had decided to build five vignettes around the combined presentation of anxiety with, respectively, physical complaints, difficult behaviour, mood, developmental and school attendance problems. On the level of the key aspect mixed-anxiety, each vignette represented symptoms related mostly to one of these domains while also depicting symptoms of anxiety that do not overlap with these mental health problems.

To theoretically support the process, we first consulted clinical handbooks and questionnaires. ${ }^{40-42}$ Relevant symptom expressions and idioms were obtained from actual clinical referral letters written by GPs of children and adolescents diagnosed in specialised MHC. This linked text-data enabled working back and forth from children's complaints mentioned by their GPs and their descriptive diagnoses, and hence facilitated a controlled development of vignettes that are also natural. The extracted descriptions were grouped into the five domains and an initial set of 11 vignettes (as advised) ${ }^{43}$ was formed. In an iterative process five vignettes were selected, further refined and for readability purposes named as V1-Somatic, V2-Behavioural, V3-Mood, V4-Developmental, V5-School Attendance. In each vignette, more cues were given for anxiety compared with any other specific disorder group (eg, in V2-Behavioural 
Table 1 Survey items as shown and verbally introduced

\section{Questions and options as shown}

A1 What is the main complaint?

\section{Questions and options verbally introduced as}

Where do you think this description fits in? To which symptom-profile would you ascribe this vignette?

1. Typical development

2. Behavioural problems

1. Option one if it is probably an example of typical development.

2. Option two for difficult behaviour: examples include aggressive behaviour or antisocial behaviour.

3. Complaints regarding establishing contact

3. If problems likely indicate an autism spectrum disorder, you can choose option three.

\section{Mood problems}

4. Mood problems and problems that could be related to depressive disorders.

\section{Somatic complaints}

5. For physical symptoms choose option five, also if a problem might be psychosomatic in nature.

6. Eating problems

7. Anxiety-related complaints

8. Complaints regarding attention and activity

6. Option six for eating problems and probable eating disorders.

7. Option seven for problems related to anxiety and anxiety disorders.

8. Option eight for attention-related complaints that might indicate attention deficit hyperactivity disorder or attention deficit disorder.

9. Complaints related to the experience of a traumatic event

9. Option nine for problems related to the experience of a traumatic event.

A2 Second complaint group?

If you would like to add a second problem to the main complaint groups you can select it here.

10. No second complaint group

10. Please choose option 10 on your voting machine if you do not see another complaint.

A3 Where should this child ideally go?

Then, for each vignette, the triage question: where can this child and the family get the most adequate professional support?

1. Practice nurse

2. Local youth teams

3. Generalised mental healthcare

4. Specialised mental healthcare

5. Somatic healthcare/hospital

Where do you think that children with this type of complaint are best helped?

B1. Behavioural problems

B2. Complaints regarding establishing contact

B3. Mood problems

B4. Somatic complaints

B5. Eating problems

B6. Anxiety-related complaints

B7. Complaints regarding attention and activity

B8. Complaints related to the experience of a traumatic event
1. Watchful waiting
2. Practice nurse
3. The local youth teams
4. General mental healthcare
5. Specialised mental healthcare

The eight mental health groups will be shown again. Please indicate for each of these how you generally tend to refer children when you suspect these complaints.
If you often tend to first monitor such complaints for a while, you can opt for ' 1 '. If you often ask your mental health practice nurse to become involved option ' 2 ', and so on, up to the point where you feel that a more intense treatment option is adequate. Then, you can choose option ' 5 ': specialised mental healthcare.

Survey items A1-A3 were presented after each vignette. Given their relevance for early recognition, the response options for the identification questions ( $\mathrm{A} 1$ and $\mathrm{A} 2$ ) were formulated in terms of 'complaints' and 'problems' rather than 'disorders'. We presented two identification questions to provide sufficient opportunity to identify an $A D$ in the mixed vignettes. The third recurring question queried how the respondents would have referred such a child or family in daily practice, and was presented with response options that reflect the Dutch 'stepped care' services. The practice nurse is a mental health professional (MHP) who works in general practice. The option 'hospital' was presented since the vignettes also depicted physical problems. As we introduced the study as one about children with psychosocial problems, the option watchful waiting was not presented for the vignettes. The survey items B1-B8 were presented after the last survey item A3. Here, the option watchful waiting was presented following our interest in whether ADs are viewed as something children grow out of.

vignette, we counted the total number of cues as 13 , with eight cues that could be related to anxiety, five to behavioural problems, three to attention-hyperactivity and trauma, two to somatic and one to autism). To mimic actual consultations, the vignettes also included contextual factors $^{43}$ such as academic or home functioning or background information about the GP-child relationship. Case age and vignette length had a limited range (10-12 years, 165-172 words) to avoid possible confounding effects. ${ }^{43}$ 
During the final stage of vignette development, GPs affiliated with Leiden University Medical Centre were invited to participate in an individual pilot interview (six interviews held in November 2018). GPs were asked to think aloud and provide verbal feedback while reading the transcript of each vignette and answering the identification and referral questions. We also asked GPs about the realism of the vignettes: they responded positively and felt that the descriptions corresponded to their practice. The pilot led to minor changes, most concerning the 'contextual aspects' ${ }^{43}$ for example, initially we alternated mothers and fathers as the parent who went along to the consultation. Several GPs evaluated this as odd, hence we changed it to 'mother'.

\section{Validation of vignettes}

To validate the recognisability of anxiety, 24 MHPs were invited to participate in a pilot presentation, of whom 11, all with extensive clinical experience (median 15-19 years), agreed to participate. All MHPs were employed at Curium-LUMC, a university-affiliated inpatient and outpatient psychiatric clinic for children and adolescents. We surveyed MHPs using the same procedure as in the GP-survey, with the exception of written responses and two additional questions regarding their psychiatric specialisation. The presentation resulted in 55 answers concerning the first identification question, and 53 answers on the second identification question. In line with our aim to develop mixed-anxiety vignettes, MHPs selected different disorder groups: anxiety $(n=22)$, $\operatorname{mood}(n=19)$, attentionhyperactivity $(\mathrm{n}=14)$, behavioural $(\mathrm{n}=12)$ and somatic $(n=10)$ problems were selected frequently, and typical development was not selected (online supplementary file A). Anxiety was the most or second most frequently selected disorder group in V1-Somatic, V2-Behavioural and V4-Developmental, and the third most frequently selected in V3-Mood and V5-School Attendance.

\section{Outcome definition and analysis}

Recognition rate was defined as the ratio of the total number of times anxiety was selected and the total number of times anxiety could have been selected. GPs' and MHPs' recognition was compared following Newcombe's method $10^{44}$ using the Epi package ${ }^{45}$ in the R-statistics environment. This method tests for independence as in the $\chi^{2}$ test, yet provides a robust method to estimate CIs. GPs' selection rate of the different disorder categories and the referral indications they made for the vignettes were analysed using descriptive statistics. Whether the likelihood of an MHC referral was lower when anxiety was recognised in a vignette was tested in a logistic multilevel regression using the lme4 package. ${ }^{46}$ That GPs would report an MHC referral for ADs less often compared with referral for behavioural, developmental and mood problems was also tested in a logistic multilevel regression, with disorder groups (anxiety vs behaviour, development and mood problems) as the repeated measures. Respondents with missing responses on more than half of the
Table 2 Demographics of the study sample and the national population

\begin{tabular}{lcll}
\hline & $\begin{array}{l}\text { Study sample } \\
\mathbf{n = 2 2 9}\end{array}$ & $\begin{array}{l}\text { Population } \\
\mathbf{n = 9 7 9 8}\end{array}$ \\
\hline Gender & & & \\
\hline Male & $116(50.7 \%)$ & $4799(49.0 \%)$ \\
Female & $95(41.5 \%)$ & $4999(51.0 \%)$ \\
Unknown & $18(7.9 \%)$ & - & \\
Experience in & & Age distribution in national \\
years & & population \\
\hline 0-2 & $4(1.7 \%)$ & $<30$ & $76(0.8 \%)$ \\
$3-5$ & $22(9.6 \%)$ & $30-34$ & $976(9.9 \%)$ \\
$6-9$ & $27(11.8 \%)$ & $35-39$ & $1428(14.6 \%)$ \\
$10-14$ & $16(7.0 \%)$ & $40-44$ & $1518(15.5 \%$ \\
$15-19$ & $35(15.3 \%)$ & $45-49$ & $1396(14.2 \%)$ \\
$>20$ & $124(54.1 \%)$ & $>50$ & $4357(44.5 \%)$ \\
\hline Unknown & $1(0.4 \%)$ & Unknown & $56(0.6 \%)$ \\
\hline
\end{tabular}

survey were excluded from all analyses. Data were visualised using the ggplot2 package. ${ }^{47}$

\section{Patient and public involvement}

Patients and/or the public were not involved in the design, or conduct, or reporting or dissemination plans of this research.

\section{RESULTS \\ Participants}

A total of 299 attendees answered at least one survey question. Of these, 239 reported being a GP. Data from 10 GPs were excluded due to missing responses. As a missing response on both identification questions of a vignette occurred 17 times, the number of times anxiety could have been selected was $1128(229 * 5-17)$.

The distribution of the sample's gender and years of experience was similar to the national population ${ }^{48}$ (table 2).

These demographics showed no association with the number of times anxiety was recognised, the referral indications GPs made for each vignette, or with their reported referral preferences for ADs (online supplementary file B).

\section{Recognition}

By selecting 167 times anxiety in the 1128 possibilities to opt for a specific disorder group, GPs reached a recognition rate of $14.8 \%$. GPs were less likely to recognise anxiety compared with MHPs (OR $=0.26,95 \%$ CI 0.15 to 0.46 , figure 1$)$. Whereas $55.9 \%(\mathrm{n}=128)$ of the GPs labelled one or more vignette as anxiety-related, over two-fifths did not in any. On average, GPs often selected behavioural, mood, developmental and trauma-related problems, and typical development (table 3). GPs and 


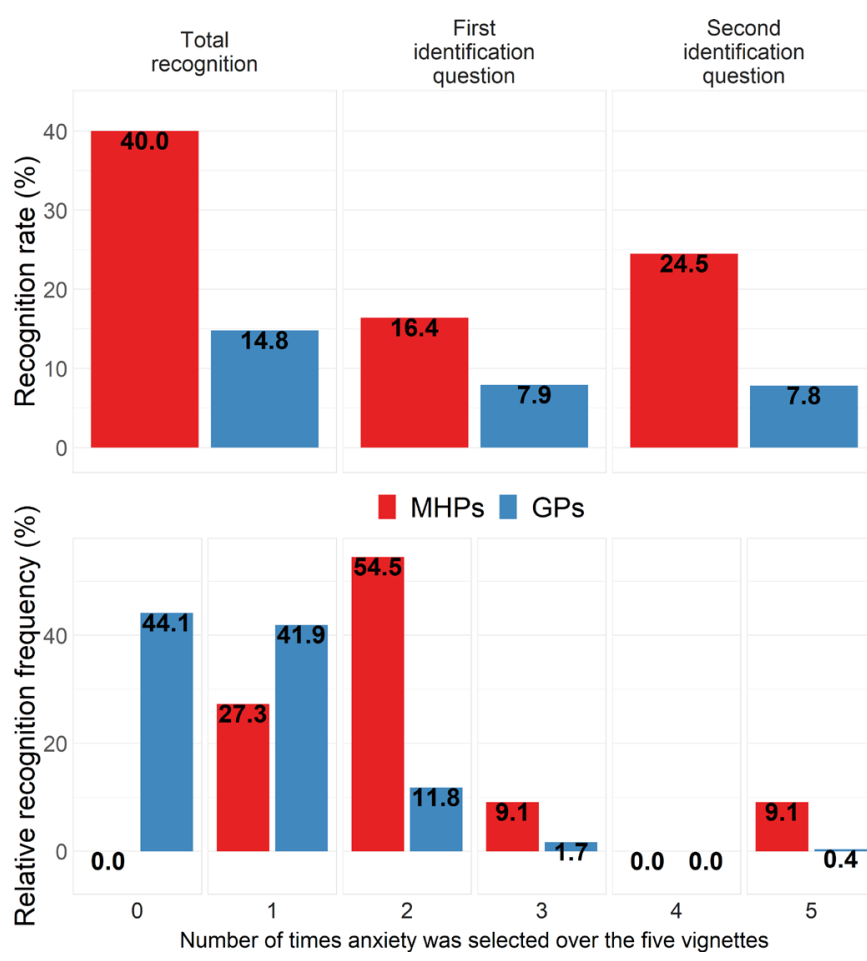

Figure 1 The upper figure depicts MHPs' and GPs' recognition rate of anxiety. MHPs selected anxiety in nine of their 55 responses on the first identification question and in 13 of their 53 responses on the second identification question. GPs selected anxiety in 84 of their 1060 responses on the first identification question and in 83 of their 1067 responses on the second identification question. Differences between the two groups were statistically significant overall (OR=0.26, $95 \% \mathrm{Cl} 0.15$ to 0.46 ), in the first identification question (OR=0.44, $95 \% \mathrm{Cl} 0.21$ to 0.93 ), and in the second identification question ( $\mathrm{OR}=0.26,95 \% \mathrm{Cl} 0.13$ to 0.50 ). The lower figure depicts the percentage of GPs and MHPs that recognised anxiety in none, one, two, three, four or all five vignettes. The difference between GPs' and MHPs' recognition frequency was statistically significant, $\chi^{2}$ ${ }_{240)}=42.94, \mathrm{p}<0.001$. GPs, general practitioners; MHPs, mental health professionals.

MHPs did not differ in their selection rate of these disorder groups (online supplementary file B).

Exploration of recognition per vignette revealed a major difference between GPs and MHPs for V1-Somatic (GPs: $5.7 \%$, MHPs: $54.5 \%$ ). Differences were not statistically significant for V2-Behavioural (GPs: 33.8\%, MHPs: $45.5 \%$ ), and for V3-Mood (GPs: $13.7 \%$, MHPs: $18.2 \%$ ). GPs recognised anxiety significantly less often than MHPs in V4-Developmental (GPs: 9.0\%, MHPs: 36.4\%) and in V5-School Attendance (GPs: 11.9\%, MHPs: 45.5\%).

\section{Referral}

When asked how they would refer a child with a similar profile to the vignettes, the majority responded that they would keep the child in general practice (practice nurse $=40 \%$, figure 2). GPs who indicated that they would refer most often chose primary healthcare (local youth teams $=24 \%$, primary $\mathrm{MHC}=13 \%$ ). The vignette which most often triggered referral to MHC was V3-Mood.
Those GPs who recognised anxiety reported referral of a child to MHC slightly less often compared with those who did not recognise anxiety (table 1 , online supplementary file B). However, multilevel analysis revealed that the likelihood of a referral to MHC was not significantly related to the recognition of anxiety $(\mathrm{OR}=0.70,95 \% \mathrm{CI}$ 0.42 to 1.18 ) (table 4 ).

When asked about their general referral preferences, a majority reported a tendency to refer children with suspected ADs to primary MHC (figure 3). More GPs reported treatment of suspected anxiety in MHC (OR=1.79, 95\% CI 1.35 to 2.37) compared with suspected developmental, behavioural or mood problems $(\mathrm{OR}=0.53,95 \% \mathrm{CI} 0.39$ to 0.72$)$. Analysis per disorder group revealed that GPs' reported referral preferences for anxiety, did not differ from their preferences for mood problems. A referral to MHC was reported less often for difficult behaviour, problems related to autism, attention-hyperactivity and (psycho-)somatic problems and more often for eating problems and trauma (online supplementary file B).

\section{DISCUSSION}

To investigate GPs' sensitivity towards ADs, we presented vignettes describing the mixture of problems that might be disclosed during consultations. GPs noticed anxiety sufficiently in the vignette that depicted a prototypic sign ('fears') but infrequently when the depicted symptoms were less explicit. Possible alternative mental health problems were recognised also by MHPs who helped to validate the vignettes but they differed from GPs only in their better recognition of anxiety. Whether a GP indicated a referral to MHC for a given vignette showed no significant association with recognition of anxiety in that vignette. MHC was selected by GPs as the appropriate referral option in less than one-fifth of these vignettes depicting problems of mild severity. Interestingly, when asked about their general referral preferences for children with suspected ADs, over two-thirds of the GPs reported a preference for intervention in MHC.

\section{Strengths and limitations of the study}

To the best of our knowledge, this is the first study to investigate GPs' interpretations of the various problems typical in children with ADs. We surveyed a large sample but we do not have insight into the representativeness of the attending GPs. Nevertheless, the years of experience and gender distribution of the sample were similar to the national GP population, these demographics did not show an association with the outcome measures. ${ }^{32}$ Also, literature is inconclusive in whether GPs' attitudes influence decision-making. ${ }^{30} 31$ The presentation of the vignettes to all participants at once yielded a risk of carryover effects. It also incited a game aspect that may have decreased observer effects and improved access to participants' honest and direct considerations. ${ }^{43} \mathrm{~A}$ limitation is the small number of MHPs and the multiple 
Table 3 GPs' selection rate of each disorder category

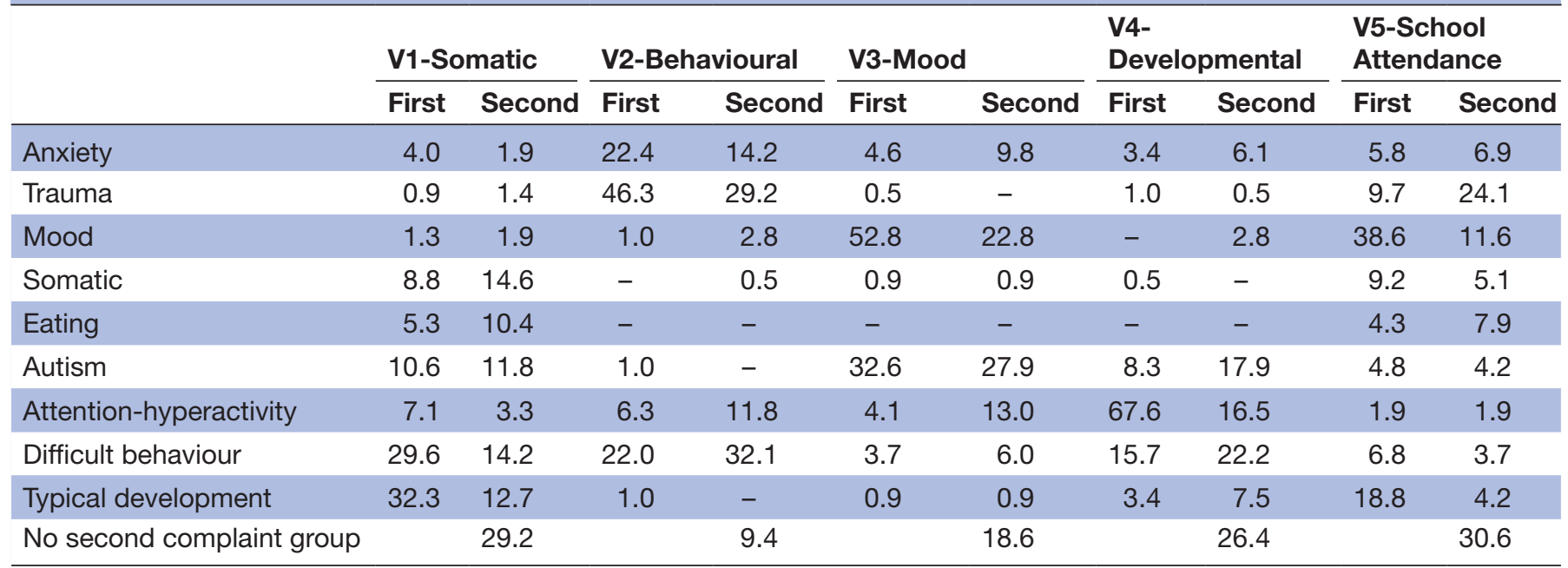

Selection rate of each disorder group shown in percentages, per identification question (first and second) and per vignette (V1-V5). Missing responses resulted in the following sample sizes in V1-Somatic=226 and 212, V2-Behavioural=205 and 212, V3-Mood=218 and 215, V4Developmental=204 and 212, V5-School Attendance=207 and 216, with the first value depicting the sample size in the first identification question, and the latter the second identification question of each vignette. GPs, general practitioners.

statistical comparisons made between GPs and MHPs. Notwithstanding the explorative nature of the study on the level of the individual vignettes, we applied conservative Bonferroni corrections to avoid false positives. MHPs selected anxiety infrequently in the mixed anxietydepression vignette. This low recognition rate might be a consequence of similar number of cues for anxiety (8) and depression (7) in this vignette, the large conceptual overlap between anxiety and depression and that MHPs might also be more attuned towards depression. ${ }^{49}$ GPs could not indicate watchful waiting for the vignettes in this study overestimating the likelihood that GPs take any action when presented with children similar to the vignettes. In conversations with GPs. we had found that in case of any ambiguity they would make the rational decision for further enquiry. Given our aim to gain insight in

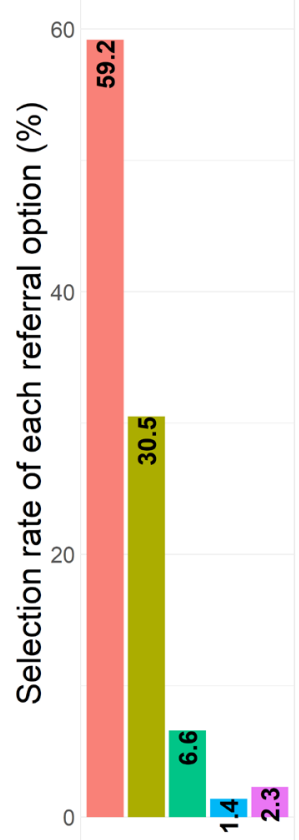
V1-
Somatic

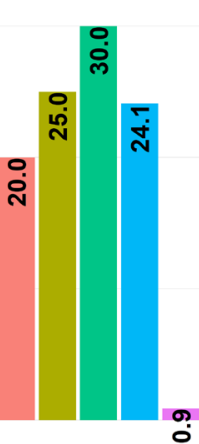

V2Behavioural

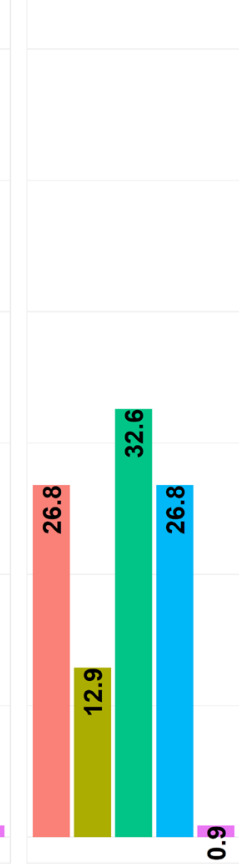

V3-Mood

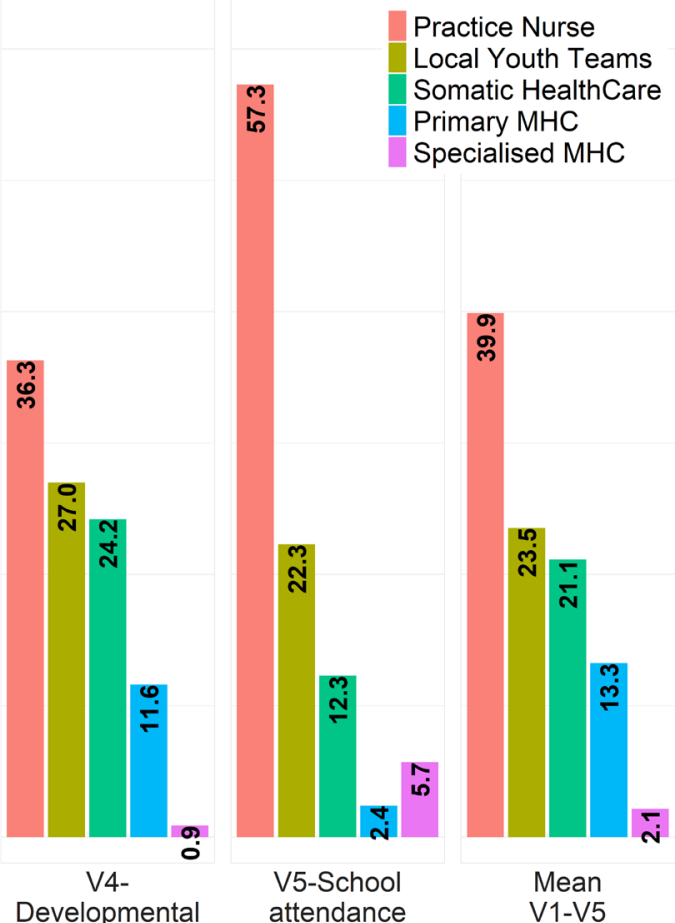

Developmental

Behavioral=220, V3-Mood=224, V4-Developmental=215, V5-School Attendance=211. GPs, general practitioners; MHPs, menta health professionals. 
Table 4 Referral to mental healthcare by those GPs who recognised anxiety and those who did not

\begin{tabular}{llcc}
\hline & Other referral & $\begin{array}{l}\text { Mental } \\
\text { options }\end{array}$ & \multicolumn{1}{c}{ healthcare } \\
\hline V1-Somatic & Selected & $13(100 \%)$ & $0(0 \%)$ \\
\hline V2-Behavioural & Selected & $59(78.7 \%)$ & $16(21.3 \%)$ \\
& Not selected & $105(73.9 \%)$ & $37(26.1 \%)$ \\
\hline V3-Mood & Selected & $24(80.0 \%)$ & $6(20.0 \%)$ \\
\hline V4- & Not selected & $137(71.0 \%)$ & $56(29.0 \%)$ \\
Developmental & Selected & $17(89.5 \%)$ & $2(10.5 \%)$ \\
& Not selected & $166(86.9 \%)$ & $25(13.1 \%)$ \\
V5-School & Selected & $22(91.7 \%)$ & $2(8.3 \%)$ \\
Attendance & Not selected & $170(91.9 \%)$ & $15(8.1 \%)$ \\
\hline Mean V1-V5 & Selected & $88.0 \%$ & $12.0 \%$ \\
\hline & Not selected & $83.9 \%$ & $16.1 \%$ \\
\hline
\end{tabular}

Selection frequency of each referral option per vignette partitioned by GPs who selected anxiety and who did not. Data revealed no significant associations between recognition of anxiety and referral to $\mathrm{MHC}(\mathrm{OR}=0.70,95 \% \mathrm{Cl} 0.42$ to $1.18, \mathrm{p}=0.19)$. An $\mathrm{MHC}$ referral includes referral to primary $\mathrm{MHC}$ and specialised $\mathrm{MHC}$, other referral options include the mental health practice nurse in general practice, local youth teams and somatic healthcare (selection rates of each specific referral option are depicted in online supplementary file B). GPs, general practitioners; MHC, mental healthcare.

their tendencies, we decided to prevent selection of this safe option. Nevertheless, it is unlikely that addition of this option would change the results considering referral to MHC. Those who would have selected watchful waiting now probably selected the practice nurse. A related caveat is the interpretation of the findings as representing recognition and referral in everyday practice. Decision-making in general practice is shaped by a complex interplay of multiple individual and systemic factors over time. With this novel approach, we aimed to isolate the extent to which GPs mind anxiety in their early diagnostic opinion. In this regard, a major strength of the study was the use of actual patient medical records to create the vignettes. This enabled wording similar to that of GPs, improving the construct and external validity of the case descriptions and diminishing uncontrollable variability. ${ }^{50}$

\section{Comparison with other studies}

Overall recognition of anxiety was within the lower range of what is found in literature $(9 \%-60 \%) .{ }^{27} 2851$ This might be a consequence of the mild severity portrayed in the vignettes, a factor limiting recognition of all disorder groups. ${ }^{32}$ This design, however, does not explain why GPs selected the other mental health problems yet failed to recognise anxiety proportionately. In V1-Somatic depicting a consultation for recurring earaches, for example, GPs picked up on the probability of behavioural, somatic or autism spectrum-related problems. They were also sensitive to whether typical development was depicted. Nonetheless, the portrayal should have also raised the prospect of an $\mathrm{AD}$, a possibility that was recognised by very few GPs. GPs recognised anxiety sufficiently in one vignette. This being the vignette that literally mentioned the core feature of anxiety ('fears') supports that unfamiliarity with symptom presentation might be the driving factor behind the low recognition rates.

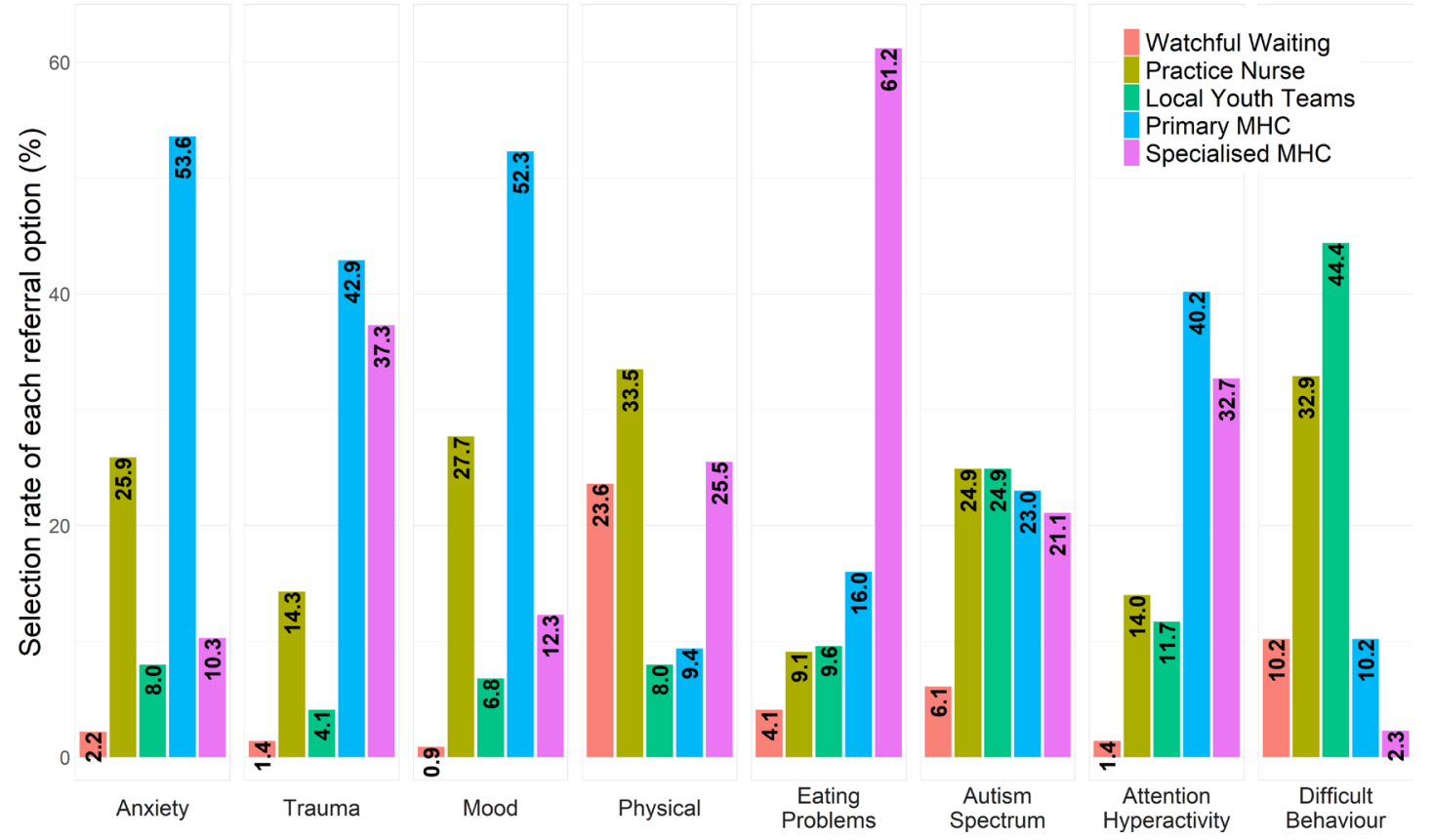

Figure 3 GPs' reported referral preferences for each of the eight disorder groups. Excluding cases with missing responses left the following sample sizes: anxiety $n=224$, trauma $n=217$, mood $n=220$, somatic $n=212$, eating problems $n=219$, autism $n=213$, attention-hyperactivity $n=214$, difficult behaviour $n=216$. GPs, general practitioners; MHC, mental health care. 
The recognition rate was realised by just over half of GPs. Others did not select anxiety in any vignette. A question is what underlies these individual differences. Previous studies also found no association of recognition with gender or experience of the professional, and literature is not one-sided about the role of attitude. ${ }^{31}$ Observations we made during the pilot with six GPs suggest that differences might be caused by how they "perceive, read and interpret ${ }^{37}$ presenting symptoms. For instance, all GPs commented about the word 'temperamental', yet many neglected 'timid' although both words were in the same sentence. GPs also varied in the problem areas they gave attention to. Most GPs expressed a focus on school and home burden (eg, 'School is a benchmark for me and that is going well. If it wasn't alarm bells would be going off'). By contrast, many did not reveal a need to enquire further with children themselves or about leisure activities and social relations. If a GP tends to piece out some symptom-profiles and neglect others based on context, ${ }^{52} 53$ this might be of particular consequence for the recognition of ADs characterised by a covert and less disruptive profile.

An important finding concerned the final vignette which portrayed a child with social $\mathrm{AD}$. In response to this vignette, GPs frequently selected typical development and the mildest referral options. Although a similar response pattern was obtained for the first vignette, both the vignette developers and MHPs evaluated the final vignette as a more severe case description. This finding is in line with studies suggesting low recognition of social anxiety. ${ }^{29} 3554$ Recognition of social anxiety by professionals is of particular importance since these patients are known to under-report symptoms, and since diagnosisspecific interventions are advised in clinical guidelines. ${ }^{55}$

Contrary to hypothesis, GPs did not prove less likely to prefer intervention in MHC for ADs compared with problems related to difficult behaviour, developmental disorders or depression. Results of this normative part of the study contrasts with clinical studies demonstrating that a substantial proportion of children with ADs are unlikely to be referred to MHC. ${ }^{25} 3056$ This disparity with clinical practice might be related to factors other than GPs' conscious evaluations, such as strain on parents and their treatment desires that differ between disorders. ${ }^{57}$ Another explanation might be a discrepancy in GPs conscious evaluations on the one hand, and their skills and implicit decisionmaking tendencies on the other hand. Our data support this explanation to some extent, although to our knowledge, no previous study examined the evaluations and implicit tendencies GPs hold towards treatment of children with ADs. We found a discrepancy in GPs' responses in that the majority of the GPs reported to consider a referral when they suspect ADs, but generally chose management in primary healthcare rather than referral to MHC in the vignettes. This comparison of reported referral preferences to the referral indications made for the vignettes might be invalid if GPs assumed a higher severity when asked about referral preferences versus the mild severity depicted in the vignettes. However, in both, we emphasised that the survey considers probable mental health problems, including subthreshold levels of severity. The variations in GPs' answers between disorder groups supports that they understood the question as it was meant. Also, GPs were inclined to refer the vignette scenario with symptoms that overlapped with mood problems to MHC, in agreement with their reported normal practice. This leaves open whether their threshold to evaluate anxiety as an $\mathrm{AD}$ differs from when they consider mood problems as depression, ${ }^{54}$ yet supports our hypothesis that differences in the likelihood of a referral may depend on symptom presentation. Notably, during the individual pilot interviews, we observed a focus on symptoms related to depression 'because you would not want to leave it untreated' and varying heuristics GPs used when evaluating the relevance of symptoms.

\section{Meaning of the study}

Moving beyond sensitivity as the endpoint of diagnostic accuracy, the results suggest that the repeatedly found underrecognition of anxiety goes back to the initial considerations GPs make. Our findings confirm the conjecture that recognition of ADs is often confounded by their overlap with other common and well-known mental health problems and similarity with typical developmental variations. ${ }^{26}$ However, this finding per se does not distinguish between the alternate possibilities that GPs prioritise other disorder groups or lack sufficient knowledge of the presentation and prevalence of child anxiety. In fact, it has been suggested that there might be a prioritisation issue given the seemingly low burden and impact of ADs. ${ }^{38}$ In the current study, the presentation of the vignettes using subtitled audio fragments precluded the possibility of rereading and elaborate evaluation. By verbally presenting vignettes that do not indicate a distinctive mental health disorder, we aimed to circumvent knowledge of diagnostic criteria and the question of a primary or differential diagnosis. The study results therefore imply that, even before rational prioritisation, there could be a significant bottleneck in first interpretation that might have otherwise led to early recognition. Anxiety might not resonate sufficiently as the likely problem when GPs encounter children with ADs. Leaving aside the discussion of whether it is a GP's task to assess the specifics of a child's mental health problem, we argue that it is worthwhile to improve knowledge of early symptom presentation in children with problems that are relatively poorly recognised by parents and teachers. GPs face the difficult task to recognise mental health problems in a limited amount of consult time, yet report difficulties with the use of structured assessment methods in clinical practice. ${ }^{58}$ To avail the benefits of their unguided decision-making, it is necessary that they become more attuned and aware of the prevalence, the breadth and relevance of typical signs of anxiety. Understanding how professionals comprehend and act on presenting symptoms is important, even more so in light of the high 
base-rate of ADs, ${ }^{8}$ their early age of onset $(11 \text { years })^{18}$ and risk of persistence into adulthood. ${ }^{1-3}$ The finding that majority of the GPs reported to prefer an MHC referral indicates that they do acknowledge anxiety as a treatment demanding problem, and leaves opportunity for targeted improvements.

\section{Unanswered questions and future research}

Why did some GPs recognise anxiety in multiple vignettes whereas others did not? Another open question concerns how GPs would have responded had the referral option 'no action needed' been available for the vignettes. Causal inferences as regards symptom presentation and subsequent recognition and referral could be made if the many symptoms associated with ADs could be systematically varied using a factorial vignette design. ${ }^{43}$ If combined with qualitative methods, future studies could further enhance our understanding of the decision-making process.

Acknowledgements We gratefully acknowledge the organisers and participants of the 14 December 2018 Boerhaave Symposium, the participants of the 27 November 2018 validation meeting with mental health professionals at CuriumLUMC Leiden, Umut Can Yildiz for his technical advices in embedding the vignette transcripts as subtitles into the audio fragments, and Dr Elise Dusseldorp for her advices regarding the multilevel analyses.

Contributors SA analysed clinical referral letters of children, created the vignette drafts and drafted the manuscript. MC, PMW, BMS and MEN provided feedback to the vignette drafts during the iterative vignette development process. MC, MW and BMS contributed to the interpretation of the data. SA, MC, BMS, RRJV, MEN and PMW designed the study, critically revised and gave final approval for the manuscript.

Funding The authors have not declared a specific grant for this research from any funding agency in the public, commercial or not-for-profit sectors.

Competing interests None declared.

Patient consent for publication Not required.

Provenance and peer review Not commissioned; externally peer reviewed.

Data availability statement Data are available upon reasonable request. No additional data are available for this study in repositories. Inquiries concerning the data may be made to the corresponding author.

Open access This is an open access article distributed in accordance with the Creative Commons Attribution Non Commercial (CC BY-NC 4.0) license, which permits others to distribute, remix, adapt, build upon this work non-commercially, and license their derivative works on different terms, provided the original work is properly cited, appropriate credit is given, any changes made indicated, and the use is non-commercial. See: http://creativecommons.org/licenses/by-nc/4.0/.

\section{ORCID iD}

Semiha Aydin http://orcid.org/0000-0002-1499-2034

\section{REFERENCES}

1 Beesdo K, Knappe S, Pine DS. Anxiety and anxiety disorders in children and adolescents: developmental issues and implications for DSM-V. Psychiatr Clin North Am 2009;32:483-524.

2 Bittner A, Egger HL, Erkanli A, et al. What do childhood anxiety disorders predict? J Child Psychol Psychiatry 2007;48:1174-83.

3 Copeland WE, Angold A, Shanahan L, et al. Longitudinal patterns of anxiety from childhood to adulthood: the great Smoky Mountains study. J Am Acad Child Adolesc Psychiatry 2014;53:21-33.

4 Haller $\mathrm{H}$, Cramer H, Lauche R, et al. The prevalence and burden of subthreshold generalized anxiety disorder: a systematic review. BMC Psychiatry 2014;14:128.

5 Woodward LJ, Fergusson DM. Life course outcomes of young people with anxiety disorders in adolescence. J Am Acad Child Adolesc Psychiatry 2001;40:1086-93.
6 Bosman RC, Ten Have M, de Graaf R, et al. Prevalence and course of subthreshold anxiety disorder in the general population: a three-year follow-up study. J Affect Disord 2019;247:105-13.

7 Pape K, Bjørngaard JH, Holmen TL, et al. The welfare burden of adolescent anxiety and depression: a prospective study of 7500 young Norwegians and their families: the HUNT study. BMJ Open 2012;2:e001942.

8 Baxter AJ, Scott KM, Vos T, et al. Global prevalence of anxiety disorders: a systematic review and meta-regression. Psychol Med 2013:43:897-910.

9 Merikangas KR, He J-P, Burstein M, et al. Lifetime prevalence of mental disorders in U.S. adolescents: results from the National Comorbidity Survey Replication--Adolescent Supplement (NCS-A). J Am Acad Child Adolesc Psychiatry 2010;49:980-9.

10 Chisholm D, Sweeny K, Sheehan P, et al. Scaling-up treatment of depression and anxiety: a global return on investment analysis. Lancet Psychiatry 2016;3:415-24.

11 Bennett K, Manassis K, Duda S, et al. Preventing child and adolescent anxiety disorders: overview of systematic reviews. Depress Anxiety 2015;32:909-18.

12 Warwick H, Reardon T, Cooper P, et al. Complete recovery from anxiety disorders following cognitive behavior therapy in children and adolescents: a meta-analysis. Clin Psychol Rev 2017:52:77-91.

13 Moreno-Peral P, Conejo-Cerón S, Rubio-Valera M, et al. Effectiveness of psychological and/or educational interventions in the prevention of anxiety: a systematic review, meta-analysis, and meta-regression. JAMA Psychiatry 2017;74:1021-9.

14 Teubert D, Pinquart M. A meta-analytic review on the prevention of symptoms of anxiety in children and adolescents. J Anxiety Dis 2011;25:1046-59.

15 Garralda E. Child and adolescent psychiatry in general practice. Aust N Z J Psychiatry 2001;35:308-14.

16 World Health Organization. The world health report: 2001 mental health: new understanding, new hope. Geneva: World Health Organization, 2001.

17 Garralda ME, Bowman FM, Mandalia S. Children with psychiatric disorders who are frequent attenders to primary care. Eur Child Adolesc Psychiatry 1999;8:34-44.

18 Kessler RC, Berglund P, Demler O, et al. Lifetime prevalence and age-of-onset distributions of DSM-IV disorders in the National comorbidity survey replication. Arch Gen Psychiatry 2005;62:593-602.

19 Gieteling MJ, Lisman-van Leeuwen Y, Passchier J, et al. The course of mental health problems in children presenting with abdominal pain in general practice. Scand J Prim Health Care 2012;30:114-20.

20 Rockhill C, Kodish I, DiBattisto C, et al. Anxiety disorders in children and adolescents. Curr Probl Pediatr Adolesc Health Care 2010;40:66-99

21 Zwaanswijk M, Verhaak PFM, van der Ende J, et al. Consultation for and identification of child and adolescent psychological problems in Dutch general practice. Fam Pract 2005;22:498-506.

22 Bayram C, Harrison C, Charles J, et al. 'The kids are alright' Changes in GP consultations with children 2000-15. Aust Fam Physician 2015;44:877-9.

23 Roberts J, Sanci L, Haller D. Global adolescent health: is there a role for general practice? Br J Gen Pract 2012;62:608-10.

24 Starline Health and health care; personal characteristics. Health and health care; general practice consults by personal characteristics, 2019. Available: https://opendata.cbs.nl/statline/\#/CBS/en/dataset/ 83005ENG/table?dl=20824

25 Hyde J, Evans J, Sharp D, et al. Deciding who gets treatment for depression and anxiety: a study of consecutive GP attenders. Br J Gen Pract 2005;55:846-53.

26 Kroenke K, Spitzer RL, Williams JBW, et al. Anxiety disorders in primary care: prevalence, impairment, comorbidity, and detection. Ann Intern Med 2007;146:317.

27 Olariu E, Forero CG, Castro-Rodriguez Jl, et al. Detection of anxiety disorders in primary care: a meta-analysis of assisted and unassisted diagnoses. Depress Anxiety 2015;32:471-84.

28 Chavira DA, Stein MB, Bailey K, et al. Child anxiety in primary care: prevalent but untreated. Depress Anxiety 2004;20:155-64.

29 Janssen EHC, van de Ven PM, Terluin B, et al. Recognition of anxiety disorders by family physicians after rigorous medical record case extraction: results of the Netherlands study of depression and anxiety. Gen Hosp Psychiatry 2012;34:460-7.

30 Steele MM, Lochrie AS, Roberts MC. Physician identification and management of psychosocial problems in primary care. $J$ Clin Psychol Med Settings 2010;17:103-15.

31 Koning NR, Büchner FL, Verbiest MEA, et al. Factors associated with the identification of child mental health problems in primary care-a systematic review. Eur J Gen Pract 2019;25:116-27. 
32 Tylee A, Walters P. Underrecognition of anxiety and mood disorders in primary care: why does the problem exist and what can be done? J Clin Psychiatry 2007;68 Suppl 2:27-30.

33 Reardon T, Harvey K, Young B, et al. Barriers and facilitators to parents seeking and accessing professional support for anxiety disorders in children: qualitative interview study. Eur Child Adolesc Psychiatry 2018;27:1023-31.

34 Neil L, Smith M. Teachers' recognition of anxiety and somatic symptoms in their pupils. Psychol Sch 2017;54:1176-88.

35 Herbert JD, Crittenden K, Dalrymple KL. Knowledge of social anxiety disorder relative to attention deficit hyperactivity disorder among educational professionals. J Clin Child Adolesc Psychol 2004;33:366-72.

36 Furnham A, Lousley C. Mental health literacy and the anxiety disorders. Health 2013;05:521-31.

37 Elstein AS, Schwartz A, Schwarz A. Clinical problem solving and diagnostic decision making: selective review of the cognitive literature. BMJ 2002;324:729-32.

38 O'Brien D, Harvey K, Young B, et al. Gps' experiences of children with anxiety disorders in primary care: a qualitative study. $\mathrm{Br} J \mathrm{Gen}$ Pract 2017;67:e888-98.

39 Aydin S, Westenberg PM, eds. Psychosocial problems in youths: What does the GP decide? Boerhaave Continuing Medical Education conference: Progress and Practice 13 and 14 December. Leiden, The Netherlands, 2018.

40 Carr A. The Handbook of child and adolescent clinical psychology: a contextual approach. Second ed. London and New York: Routledge Taylor \& Francis Group, 1999.

41 DAWBA. Dutch version of the development and well-being assessment. Available: https://www.dawba.info/py/dawbainfo/f1ned. py\#b

42 Goodman A, Heiervang E, Collishaw S, et al. The 'DAWBA bands' as an ordered-categorical measure of child mental health: description and validation in British and Norwegian samples. Soc Psychiatry Psychiatr Epidemiol 2011;46:521-32.

43 Evans SC, Roberts MC, Keeley JW, et al. Vignette methodologies for studying clinicians' decision-making: validity, utility, and application in ICD-11 field studies. Int J Clin Health Psychol 2015;15:160-70.

44 Newcombe RG. Interval estimation for the difference between independent proportions: comparison of eleven methods. Stat Med 1998;17:873-90.

45 Stevenson M. epiR: tools for the analysis of epidemiological data 2019.
46 Bates D, Mächler M, Bolker B, et al. Fitting Linear Mixed-Effects Models Using Ime4. J Stat Softw 2015;67:1-48.

47 Wickham H. ggplot2: elegant graphics for data analysis. New York: Springer-Verlag, 2016.

48 LFJvd V, Kasteleijn A, Kenens RJ. Cijfers uit de registratie van huisartsen, 2016. Available: https://nvl004.nivel.nl/nivel-2015/sites/ default/files/cijfers-uit-de-registratie-van-huisartsen-peiling-januari2016.pdf

49 Asp M, Lindqvist D, Fernström J, et al. Recognition of personality disorder and anxiety disorder comorbidity in patients treated for depression in secondary psychiatric care. PLoS One 2020;15:e0227364.

50 Elstein AS, Rovner DR, Holzman GB, et al. Psychological approaches to medical decision making. American Behavioral Scientist 1982;25:557-84.

51 Merikangas KR, He J-ping, Burstein M, et al. Service utilization for lifetime mental disorders in U.S. adolescents: results of the National comorbidity Survey-Adolescent supplement (NCS-A). J Am Acad Child Adolesc Psychiatry 2011;50:32-45.

52 Lykke K, Christensen P, Reventlow S. "This is not normal" signs that make the GP question the child's well-being. Fam Pract 2008;25:146-53.

53 Wissow LS, Zafar W, Fothergill K, et al. Using vignettes to assess contributions to the work of addressing child mental health problems in primary care. BMC Health Serv Res 2016;15:584.

54 Paulus DJ, Wadsworth LP, Hayes-Skelton SA. Mental health literacy for anxiety disorders: how perceptions of symptom severity might relate to recognition of psychological distress. J Public Ment Health 2015;14:94-106.

55 National Institute for Health and Care Excellence. Social anxiety disorder: recognition, assessment and treatment Clinical guideline [CG159], 2013. Available: https://www.nice.org.uk/guidance/cg159/ chapter/1-Recommendations\#interventions-for-children-and-youngpeople-with-social-anxiety-disorder-2

56 Wang PS, Angermeyer M, Borges G, et al. Delay and failure in treatment seeking after first onset of mental disorders in the world Health organization's world mental health survey initiative. World Psychiatry 2007;6:177-85.

57 Chavira DA, Garland A, Yeh M, et al. Child anxiety disorders in public systems of care: comorbidity and service utilization. J Behav Health Serv Res 2009;36:492-504.

58 Wittchen $\mathrm{H}-U$, Mühlig S, Beesdo K. Mental disorders in primary care. Dialogues Clin Neurosci 2003;5:115-28. 